\title{
INDEX TO VOLUME 60
}

\author{
INDEX OF ABSTRACTS
}

Subjects:

Algebra and Theory of Numbers, 22, 46, 61, 75, 135, 249, 329, 359, 386, 472, 522

Analysis, 26, 49, 63, 78, 143, 252, 337, 365, 392, 478, 533

Applied Mathematics, 32, 52, 71, 80, 164, 256, 345, 376, 394, 481, 548

Geometry, 34, 54, 72, 82, 167, 257, 349, 379, 483, 551

Logic and Foundations, 34, 72, 83, 169, 258, 350, 396, 483, 555

Statistics and Probability, 34, 73, 171, 259, 351, 381, 556

Topology, 35, 54, 73, 83, 171, 259, 351, 383, 398, 484, 558

\section{Authors:}

Abramowitz, M., 345; Addison, J. W., 258; Al-Dhahir, M. W., 551, 555; Amitsur, S. A., 135, 136; Anderson, A. G., 556; Anderson, F. W., 558; Anderson, R. D., 35, 559; Andrushkiw, J., 26; Ankeny, N. C., 136; Antosiewicz, H. A., 345, 365, 533; Apostol, T. M., 522; Arens, R., 478; Aronszajn, N., 63, 533; Arsove, M. G., 478; Artzy, R., 82, 82, 522, 551; Aucoin, C. V., 54; Auslander, M., 383, 383; Avann, S. P., 386; Ayer, M. C., 381.

Bade, W. G., 26, 26, 337; Bade, W. L., 548; Baer, R. M., 143, 143; Bagemihl, F., 27, 144; Bagley, R., 55; Barnes, W. E., 472, 472; Barrett, J. H., 49, 337; Bartle, R. G., 534; Bartholomay, A. F., 351; Baum, J. D., 171, 171 ; Baxter, G. E., 381, 556; Beaumont, R. A., 75, 475; Bechhofer, R. E., 34; Becker, H. W., 136, 137, 249, 249, 249, 250, 329, 330, 473, 473, 474, 474, 522, 523, 523; Bell, P. O., 552; Bendat, J. S., 534; Bergman, S., 144, 337, 534; Bernhart, A., 534; Bers, L., 27, 27, 32; Bing, R. H., 172, 383, 384, 559; Birkhoff, G., 524; Blackburn, J. F., 53; Blair, R. L., 474; Blake, R. G., 548; Blank, A. A., 376; Bledsoe, W. W., 252, 535; Block, H. D., 377, 377, 535; Boas, R. P., Jr., 28, 535; Bohnenblust, H. F., 80; Bott, R., 172; Botts, T. A., 475; Bourgin, D. G., 28; Brace, J. W., 172; Brauer, A. T., 46, 330, 330; Bremermann, H. J., 392, 392, 392; Brenner, J. L., 331, 359, 359, 359, 386, 475; Briggs, W. E., 359; Browder, F. E., 28, 64, 144; Brown, M., 351; Brownell, F. H., 28, 256, 346; Bruck, R. H., 61, 524; Brunk, H. D., 73, 381, 535; Burgess, C. E., 173, 398; Burrow, M. D., 331; Butzer, P. L., 337; Byrne, J. R., 475.

Calabi, E., 167, 168; Calabi, L., 35; Calderon, A. P., 478; Cameron, R. H., 145, 338; Capel, C. E., 54; Carlitz, L., 22, 22, 22, 23, 46, 46, 47, 47, 47, 137, 137, 137, 250, 250, 251, 331, 332, 332, 524, 524; Carson, R. C., 145; Carter, W. C., 164; Case, J. H., 173; Caton, W. B., 366; Cesari, L., 366; Chang, Chen-chung, 76, 76, 76, 387, 396, 524; Charnes, A., 33; Chen, K. T., 61; Chen, Y. W., 338; Childress, N. A., 553; Choquet, G., 64; Chowla, S., 359; Chung, K. L., 171, 171; Churchill, R. V., 548; Civin, P., 536, 536; Clark, C. J., 387; Clifford, A. H., 138, 138; Cohn, Harvey, 61, 525; Collins, H. S., 55; Cooke, K. L., 331, 536; Cotlar, M., 145, 145, 145, 146; Cowell, W. R., 525; Coxeter, H. S. M., 349; Crouch, R. B., 525; Cunningham, F., Jr., 146; Curtis, C. W., 61; Curtis, H. J., 384; Curtiss, J. H., 381, 387.

Darsow, W. F., 360; Davis, A. C., 387; Davis, C., 146; Davis, M. D., 169; Davis, P., 536; Davis, R. B., 29, 146; Davis, R. M., 338; Day, M. M., 478, 537; Deal, R. B., 72; DeCicco, J., 168, 379, 380, 380, 380, 552; Dekker, D. B., 483; Dekker, J. C. E., 72; Dempster, A. P., 34; Dengler, M. A., 393; Deskins, W. E., 360, 360; Diaz, J. B., 346, 478; Dickinson, D. J., 29; Diliberto, S. P., 78; DiPrima, R. C., 164; Dirac, G. A., 173; Divinsky, N. J., 62; Doberly, V. J., 23, 252; Donoghue, W. F., 537; Douglas, J., Jr., 549; Doyle, T. C., 549; Duffin, R. J., 537; Dunford, N., 147; Duren, W. L., Jr., 366; Dyer, E., 351, 559. 
Eberlein, W. F., 138, 366; Edrei, A., 147, 339; Ehrlich, G., 138; Eilenberg, S., 173; Elliott, J., 147; Ellis, D. O., 55, 55, 174, 526; Epstein, M. P., 77; Erdös, P., 147, 171, 526, 526, 537, 538; Etter, D. O., 55; Evans, R. L., 71; Ewing, G. M., 381, 535.

Fadell, E. R., 352; Faucett, W. M., 55; Federer, H., 252, 339; Feeney, W. J., 170; Fekete, M., 29, 148; Feldman, J., 479; Fialkow, A., 256, 256; Ficken, F. A., 52, 148; Firey, W. J., 78; Flanders, H., 82, 139; Fleming, W. H., 367; Fletcher, H. J., 78; Floyd, E. E., 149; Ford, G. W., 174; Ford, L. R., Jr., 174; Forsythe, G. E., 476; Fort, M. K., Jr., 54, 56; Foster, A. L., 332; Fourés, Y., 148; Fox, W. C., 174; Fraïssé, R., 396, 396; Frank, E., 538; Fredrickson, E. L., 481; Friedman, H. D., 36; Friedrichs, K. O., 394; Fulks, W. B., 538; Fullerton, R. E., 367.

Gabriel, R. F., 339; Gaddum, J. W., 552; Gaier, D., 339; Gál, I. S., 36; Gambill, R. A., 64, 367; Garabedian, P. R., 65; Gass, S. S., 53; Gerst, I., 256, 256; Getoor, R. K., 557, 557; Giever, J. B., 559; Gillette, E. F., 168; Gillman, L., 144, 361, 361, 526, 526; Ginsburg, S., 50, 259; Goheen, H. E., 361; Goldberg, K., 332; Goldberg, M., 553; Goldberg, S. I., 62, 251; Goldhaber, J. K., 37; Golomb, M., 147, 538; Gomory, R. E., 340; Good, R. A., III, 47; Goodman, A. W., 65, 149; Gordon, L. I., 65; Gosselin, R. P., 368; Gras, E. C., 340, 539; Graves, L. M., 368, 368; Green, L. W., 169; Griffin, J. S., Jr., 55, 175; Griffith, H. C., 56, 384; Guy, W. T., Jr., 149, 539.

Haas, V. B., 149; Hadlock, E. H., 47; Hadnot, B. F., 50; Hailperin, T., 34; Haimo, F., 139, 361; Hale, J. K., 65, 66, 367; Hall, D. W., 47; Hall, M., 139; Halperin, I., 66; Hammer, P. C., 72, 377, 378, 553; Hamstrom, M. E., 35, 175; Hanf, W., 526; Harrold, O. G., 384; Haynsworth, E. V., 139; Heins, M. H., 150, 150; Helgason, S., 340, 341; Heller, G. I., 352; Henkin, L. A., 387; Henrici, P., 341; Henriksen, M., 175, 361, 361, 526, 526; Herstein, I. N., 23, 333, 362; Herwitz, P. S., 50; Herzog, F., 537, 538; Hewitt, E., 388; Higman, D. G., 333, 333, 334; Hille, E., 150; Hintikka, K. J. J., 350; Hirschman, I. I., Jr., 150, 369; Hirzebruch, F. E. P., 176; Hoffman, A. J., 151, 151, 169; Hoffman, W. C., 378; Holladay, J. C., 151; Hollingsworth, J., 377, 378; Horvay, G., 32; Hsiung, C. C., 349, 349; Hu, S. T., 484, 560; Hubert, A. O., 30, 34; Hudson, G. E., 80; Huff, C. W., 48; Hunt, B., 476; Hutcherson, W. R., 553, 554; Hyers, D. H., 394; Hyman, M. A., 152, 165.

Ikenberry, E., 382; Isbell, J. R., 23, 152, 259, 259.

Jaeger, A., 62, 140, 251; James, R. D., 388, 479; Jehle, H., 548; Jenner, W. E., 140, 527; Jerison, M., 369; Jerrard, R. P., 379; Johnson, G., Jr., 539, 539; Johnson, R. D., Jr., 36; Johnston, E. R., 369; Jones, F. B., 176, 560; Jonsson, B., 24, 24, 527; Juncosa, M. L., 152; Jurkat, W. B., 66, 67, 152, 153, 341.

Kadison, R. V., 252, 479; Kalicki, J., 83; Kaplansky, I., 527; Kasner, E., 380; Kaufman, H., 348; Kazarinoff, N. D., 153, 369; Keesee, J. W., 176; Kelly, J. B., 140; Kemperman, J. H. B., 153, 382; Kiefer, J., 35; Klamkin, M. S., 141; Klee, V. L., 83, 149, 483, 484, 540; Kleene, S. C., 258; Klein, G., 150; Kleinecke, D. C., 540, 540, 541; Klimczak, W. J., 334; Knopp, K., 67; Knothe, H., 554; Koch, R. J., 56, 56; Koehler, F., 153; Korevaar, J., 67, 68, 370, 541, 541, 542; Krabbe, G. L., 479, 542; Krakowski, M., 33; Kruskal, M. D., 351; Krzywoblocki, M. Z., 68, 370; Kuhn, Harold W., 36.

LaBorde, H. T., 330; Lambek, J., 335, 527; Landau, H. G., 382; Lane, R. E., 68, 154; Lang, S., 141; LaSalle, J. P., 154; Lashof, R. K., 260; Latta, G. E., 80 ; Leader, S., 393; Leavitt, W. G., 362; Ledley, R. S., 378; Lee, H. L., 48; Lee, R., 482; Lee, T. H., 48; deLeeuw, K., 335; Lehman, A. B., 176; Lehmer, D. H., 476; Lehmer, E., 476; Leipnik, R. B., 154, 542; LeVeque, W. J., 528; Levin, J. J., 68, 155; Levinson, N., 68; Levitzki, J., 24; Levy, G., 528; Linis, V., 342; Linstone, H. A., 543; Livesay, G. R., 384; Lohwater, A. J., 253, 480, 543; Lorch, E. R., 155, 253; Lorentz, G. G., 69; Loud, W. S., 370, 378; Lovaglia, A. R., 393; Ludford, G. S. S., 346, 478; Luke, Y. L., 379. McAdams, H. T., 557; McAuley, L. F., 57, 352; McKay, J. H., 388; McKelvey, R. W., 155, 369; McLeod, E. B., Jr., 371; McMillan, B., 558; McShane, E. J., 371; 
MacKenzie, R. E., 143; Mackie, A. G., 80; MacLane, G. R., 156; MacLane, S., 173; MacNerney, J. S., 50, 51, 480; MacNeille, H. M., 156; Maier, E. A., 477; Mann, W. R., 53; Marcus, M. D., 79; Markus, L., 156; Marsh, D. C. B., 362; Marx, I., 165, 371; Maschler, M., 394; Mattuck, A., 335; Maud, F. E., 81; Maximon, L. C., 346; Merkes, E. P., 372, 372; Meyer, J.-P., 560; Mihalek, R. J., 364, 528; Milkman, J., 156; Miller, K. S., 69, 257; Miller, W. S., 398; Mitchell, J., 372; Mohat, J. T., 560; Monahan, J. H., 157; Montgomery, D., 484; Moore, C. N., 157, 342; Morawetz, C. S., 346; Morelock, J. C., 554; Morse, A. P., 535; Moser, J., 342; Moser, L., 527, 528, 529; Mostert, P. S., 177, 561; Muhly, H. T., 362; Murray, F. J., 481; Myers, S. B., 372 .

Nachbin, L., 343, 373; Nash, J., 157, 165, 480; Nehari, Z., 373; Nering, E. D., 81; Nelson, D., 72, 589; Neumann, B. H., 141; Newman, M., 169; Niven, I., 388, 477; Nohel, J. A., 51; Norman, R. Z., 177; Norris, M. J., 252; Norton, F. A., 57; Novikoff, A. B., 157; Nunke, R. J., 363, 384.

Oberbeck, T. E., 77; Ohtsuka, M., 51; Olson, F. R., 25, 47, 49; O’Neill, B., 398; Osborn, H., 373; Ostrom, T. G., 554.

Paige, L. J., 141, 524; Pan, T. K., 257; Papas, C. H., 395; Parker, W. V., 49; Payne, L. E., 81, 158, 253, 257, 374, 549; Peaceman, D. W., 550; Pennington, R. H., 166, 166; Peterson, F. P., 485; Peterson, R. P., 75, 259; Petty, C. M., 54; Peyerimhoff, A., 343; Philipson, L. L., 543; Phillips, R. S., 158, 253, 254; Pierce, R. S., 524; Piranian, G., 537, 538, 543; Pitcairn, J., 177, 178; Plotnick, S. I., 166; Plunkett, R. L., 178; Pohrer, R. G., 49; Pollak, H. O., 536; Porcelli, P., 63, 374, 374; Poritsky, H., 379; Posey, E. E., 384; Potts, D. H., 80, 544; Protter, M. H., 158, 550; Punga, V., 350, 554; Pursell, L. E., 73.

Rabson, G., 369; Rachford, H. H., Jr., 550; Rall, L. B., 482; Rattray, B. A., 178; Rechard, O. W., 544; Redheffer, R. M., 79, 79; Ree, R., 389; Reich, E., 79, 79; Reichelderfer, P. V., 544; Reid, W. T., 375, 381, 535; Resch, D., 161; Ribeiro, H. B., 529; Ritt, R. K., 159; Ritter, I. F., 33, 33; Roberts, R. A., 57; Robinson, L. B., 159; Robinson, L. V., 375; Robinson, R. M., 30; Rogers, J. C., 178; Rose, G. F., 252; Rosenberg, A., 363, 529, 530; Rosenbloom, P. C., 159, 343; Rosenlicht, M. A., 141; Roth, J. P., 179; Royster, W. C., 52; Rubin, Hanan, 159; Rubin, Herman, 76, 389, 397; Rudin, M. E., 179, 353; Rudin, W., 160, 160, 343, 544, 545, 545; Ryser, H. J., 139.

Saalfrank, C. W., 37 ; Saaty, T. L., 53; Salzer, H. E., 71; Saltzer, C., 347; Samelson, H., 353, 484; Sanderson, D. E., 385; San Soucie, R. L., 389; Sard, A., 30; Schafer, R. D., 25; Schenkman, E. V., 142, 336, 530; Schneider, H., 25; Schoenberg, I. J., 160; Schoenfield, J. R., 350; Schuster, S., 34, 173; Schwartz, J., 147, 161; Schweitzer, A. R., 63, 363, 483, 555; Scott, D., 83, 389, 390; Sedney, R., 347; Seelbinder, B. M., 46; Segal, I. E., 31, 148; Seidel, W., 27; Sensenig, C. B., 349; Serrin, J. B., 161, 344; Shapiro, J. M., 338; Shapiro, N., 258; Shapiro, V. L., 31, 69, 254, 344; Shenitzer, A., 336; Sherman, S., 534; Shields, A. L., 52, 161, 545; Shiffman, M., 65; Shimada, N., 179, Shipman, J. S., 348, 348; Silverman, E., 381; Silverman, R. J., 375; Singer, I. M., 545; Singh, V., 480, 546; Sklar, A., 522, 530; Slaby, H. T., 61; Smith, K. T., 546; Sobczyk, A., 530, 546, 555, 555; Sobel, M., 31, 34; Spencer, D. E., 167, 550; Spiess, F. N., 32; Spitzer, F. L., 558; Springer, G., 161; Stark, J. M., 162; Stein, S., 84; Sternberg, R. L., 347, 348, 348; Stewart, F. M., 344; Stoker, J. J., 258; Stone, W. M., 482; Storvick, D. A., 481; Straus, E. G., 169, 394; Strother, W. L., 58; Sugar, A. C., 397, 556; Suppes, P. C., 81, 397, 398; Sussman, I., 77, 531; Swift, J. D., 390; Swingle, P. M., 58, 260, 561; Szegö, G., 162, 395.

Taam, C. T., 162, 162, 163, 344; Tarski, A., 76, 78, 83, 142, 390, 391, 391, 531; Taussky, O., 169; Thale, J. S., 32; Thomas, J. M., 52; Thompson, G. L., 179; Thorne, C. J., 78, 81; Thron, W. J., 480, 546; Thurston, W. B., 348; Titt, E. W., 546; Titus, 
C. J., 375; Tomber, M. L., 25; Tornheim, L., 363, 531; Trent, H. M., 561. 561.

Uehara, H., 179; Uhlenbeck, G. E., 174; Ullman, J. L., 163, 376; Utz, W. R., 83,

Van Tuyl, A. H., 167.

Wagner, D. H., 477; Wall, H. S., 70; Wallace, A. D., 58, 485, 485, 531; Walsh, J. L., 345; Walter, J. H., 364; Wang, H., 170; Ward, L. E., Jr., 84, 485; Ward, M., 391; Wasow, W. R., 395; Waterman, D., 70, 163; Weinberger, H. F., 158, 253, 348, 374, 550; Weiner, L. M., 63, 364, 532; Weinstein, A., 254, 482; Weisfeld, M., 142, 336; Weisner, L., 32; Weiss, E., 143; Wendel, J. G., 255; Wermer, J., 164, 545; Whaples, G., 143; Whiteman, A. L., 532; Whitehead, G. W., 486, 486; Wiegmann, N. A., 477; Wilansky, A., 255; Wilcox, L. R., 364, 528, 532; Willcox, A. B., 336; Williams, R. F., 180; Williamson, J. H., 255; Willoughby, R. A., 49; Winet, M., 81; Wisner, R. J., 389; Wolf, F., 345; Wolfe, P. S., 391; Wolfowitz, J., 35; Wolfson, K. G., 251, 532; Wolk, E. S., 37; Wright, F. M., 70, 164, 376, 547; Wyman, M., 528, 529.

Yang, C. T., 73, 385, 385; Yen, T., 364; Yood, B., 481; Yos, J. M., 551; Young, D. M., 152, 345; Young, L. C., 547, 547, 547.

Zadeh, L. A., 69; Zassenhaus, H., 25; Zelinsky, D., 365, 529, 530; Zeller, K., 255, 255 ; Zemmer, J. L., 365 ; Zink, R. E., 70; Zuckerman, H. S., 388; Zygmund, A., 162.

\section{INDEX OF RESEARCH PROBLEMS}

Bellman, R., 290, 290, 291, 291, 501, 501, 501, 501; Boas, R. P., Jr., 97, 97, 97 ; Butzer, P. L., 290.

Goffman, C., 289.

Hille, E., 96.

Johnson, R. E., 96.

Paige, L. J., 289.

Rudin, W., 399, 399, 589, 589.

Shields, A., 589, 589; Sobczyk, A., 289.

Taussky, O., 289, 290; Thrall, R. M., 97, 97.

Young, L. C., 290.

INDEX OF REPORTS OF MEETINGS AND MISCELLANEOUS ARTICLES

Borel, A. See Gleason, A. M.

Cohen, L. W. Reports of meetings of the American Mathematical Society: October Meeting in New York, 20; Annual Meeting in Baltimore, 124; February Meeting in New York, 248; April Meeting in New York, 329.

Curtis, C. W. See Gleason, A. M.

Dressel, F. G., and Youngs, J. W. T. Reports of meetings of the American Mathematical Society: April Meeting in Chicago, 354.

Gleason, A. M., Borel, A., Curtis, C. W., and Kaplansky, I. The First Summer Mathematical Institute, 457.

Green, J. W. Reports of meetings of the American Mathematical Society: November Meeting in Pasadena, 75; May Meeting in Yosemite, 386; June Meeting in Portland, 472.

Kaplansky, I. See Gleason, A. M.

Lennes, N. J. See Montgomery, D.

Meder, A. E., Jr. Report of the Treasurer, 563.

Montgomery, D., and Veblen, O. Nels Johann Lennes, 264.

Puckett, W. T. Reports of meetings of the American Mathematical Society: Summer Meeting in Laramie, 516.

Szász, O. See Szegö, G.

Szegö, G. Otto Szasz, 261. 
Veblen, O. See Montgomery, D.

Weyl, F. J. The NRC-AMS Conference on Training in Applied Mathematics, 38.

Weyl, F. J., and Youngs, J. W. T. Reports of meetings of the American Mathematical Society: November Meeting in Evanston, 59

Whyburn, W. M., Reports of meetings of the American Mathematical Society: November Meeting in Spartanburg, 45.

Youngs, J. W. T. See Dressel, F. G., Weyl, F. J.

\section{INDEX OF BOOK REVIEWS}

Arens, R. See Loomis, L. H.

Atti del Quarto Congresso dell'Unione Matematica Italiana tenuto in Taormina nei giorni 25-31 Ottobre 1951, 288.

Bateman, H. See Erdélyi, A.

Bateman, P. T. See Bohr, H.

Bellman, R. Stability theory of differential equations. F. Haas, 400. See Flügge-Lotz, I.

Bianchi, L. Opere. Vol. 1, part 2, 288. Opere. Vol. 2. Applicabilitd e problemi di deformazione, 414.

Birkhoff, G., and MacLane, S. A survey of modern algebra. Rev. ed., 94.

Blumenthal, L. M. Theory and applications of distance geometry. J. Haantjes, 272.

Boas, R. P., Jr. See Journal d'Analyse Mathématique, Mathematica Scandinavica, The Michigan Mathematical Journal, Nordisk Matematisk Tidskrift.

Bochner, S. See Yano, K.

Bohr, H. Collected mathematical works. Ed. by E. Fфlner and B. Jessen. P. T. Bateman, 576.

Boothby, W. M. See Yano, K.

Bowman, F. Introduction to elliptic functions with application. F. G. Tricomi, 587.

Buchholz, H. Die konfluente hypergeometrische Funktion mit besonderer Berilcksichtigung ihrer Anwendungen. A. Erdélyi, 185.

Busemann, H. See Sen, R. N.

Caratheodory, C. Conformal representation. Z. Nehari, 281. Theory of functions of a complex variable. Trans. by F. Steinhardt, 588.

Chandrasekharan, K., and Minakshisundaram, S. Typical means. B. Kuttner, 85.

Charrueau, A. Complexes linéaires. Faisceaux de complexes linéaires. Suites et cycles de complexes linéaires conjugues. A. T. Schafer, 283.

Chevalley, C. The algebraic theory of spinors. J. Dieudonne, 408.

Chung, K. L. See Doob, J. L.

Coddington, E. A. See Hayashi, C.

Colloque sur les fonctions de plusieurs variables, 587.

Contributions to the theory of games. Vol. 2. Ed. by H. W. Kuhn and A. W. Tucker. J. Wolfowitz, 90.

Courant, R., and Hilbert, D. Methods of mathematical physics, Vol. I. A. Weinstein, 578.

Coxeter, H. S. M. See Fejes T6th, L.

Curry, H. B. See Rosser, J. B.

Curtis, M. L. See Hilton, P. J.

Davis, P. See Sikkema, P. C.

Dieudonne, J. See Chevalley, C.

Dini, U. Opere. Vol. 1, 288.

Doob, J. L. Stochastic processes. K. L. Chung, 190.

Erdélyi, A. See Buchholz, H., Snow, C., Tables of integral transforms, Tricomi, F. G.

Erdelyi, A., Magnus, W., Oberhettinger, F., and Tricomi, F. G. Higher transcendental 
functions, Based, in part, on notes left by Harry Bateman and compiled by the Staff of the Bateman Manuscript Project. G. Szegö, 405.

Fejes Toth, L. Lagerungen in der Ebene, auf der Kugel und im Raum. H. S. M. Coxeter, 202.

Fichera, G. See Picone, M.

Flügge, W. Four-place tables of transcendental functions, 414.

Flügge-Lotz, I. Discontinuous automatic control. R. Bellman, 401.

F $\phi$ lner, E. See Bohr, H.

Forsythe, G. E. See Householder, A. S.

Fréchet, M. Pages choisies d'analyse générale. T. H. Hildebrandt, 572.

Friedman, B. See Lyttleton, R. A.

Friedrichs, K. O. Mathematical aspects of the quantum theory of fields. I. E. Segal, 575.

Fuchs, W. H. J. See McLachlan, N. W., Rothe, R.

Funk, P., Sagan, H., and Selig, F. Die Laplace-Transformation und ihre Anwendung. D. V. Widder, 286.

Gál, I. N. See Tarski, A.

Gauss, C. F. Recherches arithmétiques. Trans. by A. C. M. Poullet-Delisle, 94.

Goodman, A. W. See Kaplan, W.

Haantjes, J. See Blumenthal, L. M.

Haas, F. See Bellman, R.

Halmos, P. R. See Wang, H., Zaanen, A. C.

Hasse, H. Mathematik als Wissenschaft, Kunst und Macht. R. L. Wilder, 181.

Hayashi, C. Forced oscillations in non-linear systems. E. A. Coddington, 579.

Heins, M. See Saks, S.

Hilbert, D. See Courant, R.

Hilton, P. J. An introduction to homotopy theory. M. L. Curtis, 182.

Hildebrandt, T. H. See Fréchet, M.

Hirschman, I. I., Jr. See Lorentz, G. G.

Householder, A. S. Principles of numerical analysis. G. E. Forsythe, 488.

Howarth, L. See McShane, E. J.

Jessen, B. See Bohr, H.

Journal d'Analyse Mathématique. R. P. Boas, Jr., 285.

Kaplan, W. A first course in functions of a complex variable. A. W. Goodman, 276.

Kelley, J. L. See McShane, E. J.

Kendall, D. G. See Mann, H. B.

Kuhn, H. W. See Contributions to the theory of games.

Kuratowski, C. Topologie. Vol. 2, 94.

Kuttner, B. See Chandrasekharan, K.

Loève, M. See Munroe, M. E.

Lorch, E. R. See Picone, M.

Loomis, L. H. An introduction to abstract harmonic analysis. R. Arens, 279. See Rogosinski, W. W.

Lorentz, G. G. Bernstein polynomials. I. I. Hirschman, Jr., 277.

Lyttleton, R. A. The stability of rotating liquid masses. B. Friedman, 497.

McLachlan, N. W. Complex variable theory and transform calculus. W. H. J. Fuchs, 402.

McNaughton, R. See Wang, H.

McShane, E. J., Kelley, J. L., and Reno, F. V. Exterior ballistics. L. Howarth, 274.

McVittie, G. C. See Truesdell, C.

Maak, W. Darstellungstheorie unendlicher Gruppen und fastperiodische Funktionen. I. E. Segal, 569.

MacLane, S. See Birkhoff, G.

Magnus, W. See Erdélyi, A. 
Mann, H. B. Introduction to the theory of stochastic processes depending on a continuous parameter. D. G. Kendall, 582.

Mathematica Scandinavica, R. P. Boas, Jr., 92.

The Michigan Mathematical Journal. Vol. 2, no. 1. R. P. Boas, Jr., 586.

Minakshisundaram, S. See Chandrasekharan, K.

Mostowski, A. See Tarski, A.

Munroe, M. E. Introduction to measure and integration. M. Loève, 89.

Nehari, Z. See Caratheodory, C.

Nordisk Matematisk Tidskrift. R. P. Boas, Jr., 93.

Northcott, D. G. Ideal theory. M. Rosenlicht, 282.

Oberhettinger, F. See Erdélyi, A.

Paige, L. J. See Simultaneous linear equations and the determination of eigenvalues.

Pérès, J. Mécanique générale. C. Truesdell, 286.

Petrovskij, I. G. Vorlesungen über die Theorie der Integralgleichungen, H. Pollard, 288.

Picone, M., and Fichera, G. Trattato di analisi matematica. Vol. 1. E. R. Lorch, 574.

Pollard, H. See Petrovskij, I. G.

Poullet-Delisle, A. C. M. See Gauss, C. F.

Premier colloque sur les équations aux dérivées partielles, 588.

Reno, F. V. See McShane, E. J.

Robinson, R. M. See Tarski, A.

Rogosinski, W. W. Volume and integral. L. H. Loomis, 88.

Rosenlicht, M. See Northcott, D. G.

Rosser, J. B. Logic for mathematicians, H. B. Curry, 266.

Rothe, R., and Szabo, I. Höhere Mathematik für Mathematiker, Physiker, Ingenieure. Part VI: Integration und Reihenentwicklung im Komplexen. Gewöhnliche und partielle Differentialgleichungen. W. H. J. Fuchs, 494.

Sagan, H. See Funk, P.

Saks, S., and Zygmund, A. Analytic functions. Trans. by E. J. Scott. M. Heins, 495.

Schafer, A. T. See Charrueau, A.

Scott, E. J. See Saks, S.

Segal, I. E. See Friedrichs, K. O., Maak, W.

Selig, F. See Funk, P.

Sen, R. N. A course of geometry. H. Busemann, 287.

Sikkema, P. C. Differential operators and differential equations of infinite order with constant coefficients. P. Davis, 586.

Simultaneous linear equations and the determination of eigenvalues. Ed. by L. J. Paige and O. Taussky, 414.

Snow, C. Hypergeometric and Legendre functions with applications to integral equations of potential theory. A. Erdélyi, 580.

Steinhardt, F. See Carathéodory, C.

Symposium sobre algunos problemas matematicas que se están estudiando en Latino América. Punta del Este, 19-21 Diciembre, 1951, 95.

Szab6, I. See Rothe, R.

Szegö, G. See Erdélyi, A.

Table of $\arctan x, 94$.

Table of natural logarithms for arguments between zero and five to sixteen decimal places, 414.

Tables of circular and hyperbolic sines and cosines for radian arguments, 414.

Tables of integral transforms. Prepared under the direction of A. Erdélyi. D. V. Widder, 491.

Tables, of $10^{x}, 414$.

Tarski, A., Mostowski, A., and Robinson, R. M. Undecidable theories. I. N. Gál, 570. 
Taussky, O. See Simultaneous linear equations and the determination of eigenvalues.

Thompson, A. J. Logarithmetica Britannica, being a standard table of logarithms to twenty decimal places of the number 10,000 to 100,000, 188 .

Tricomi, F. G. Lezioni sulle funzioni ipergeometriche confluenti. A. Erdélyi, 185. See Bowman, F., Erdélyi, A.

Truesdell, C. Vorticity and the thermodynamic state in a gas flow. G. C. McVittie, 493. See Pérès, J.

Tucker, A. W. See Contributions to the theory of games.

Wang, H., and McNaughton, R. Les systèmes axiomatiques de la théorie des ensembles. P. R. Halmos, 93.

Wave motion and vibration theory, 588.

Weinstein, A. See Courant, R.

Widder, D. V. See Funk, P., Tables of integral transforms.

Wilder, R. L. See Hasse, H.

Wolfowitz, J. See Contributions to the theory of games.

Yano, K., and Bochner, S. Curvature and Betti numbers. W. M. Boothby, 404.

Zaanen, A. C. Linear analysis, P. R. Halmos, 487.

Zygmund, A. See Saks, S.

\section{INVITED ADDRESSES ${ }^{1}$}

Bellman, R. The theory of dynamic programming, 503.

- 516.

Borel, A., 248.

Erdös, P., 21.

Householder, A. S., 45.

Generation of errors in digital computation, 234.

James, R. D., 516.

Klee, V. L., 472.

Kleene, S. C., 355.

Leontieff, W., 126.

Mathematics in economics, 215.

Loewner, C., 516.

Lonseth, A. T. Approximate solutions of Fredholm-type integral equations, 415.

Massey, W. S. Some new algebraic methods in topology, 111.

Mautner, F. I., 329.

von Neumann, J., 329.

Pettis, B. J., 45.

Phillips, R. S., 516.

Pollard, H., 329.

Rosenbloom, P. C., 60.

Schiffer, M. M., 75.

- Variation of domain functionals, 303.

Snapper, E. Equivalence relations in algebraic geometry, 1.

Wallace, A. D., 126.

Waterman, A. T., 126.

The National Science Foundation Program in Mathematics, 207.

Wendel, J. G., 516.

White, P. A., 386.

Regular convergence, 431.

Widder, D. V., 126.

The convolution transform, 444.

${ }^{1}$ Where the title of the address is not given, the reference is to the Report of the Meeting at which the address was given. 